\title{
Three-dimensional Simulation Function of MATLAB in the Teaching Practice of Higher Mathematics
}

\author{
Jielin Shang ${ }^{1, \text { a }}$, Hongmei $\mathrm{Pei}^{2, \mathrm{~b}}$ and Simin Wang ${ }^{3, \mathrm{c}}$ \\ ${ }^{1}$ Department of Basis, Dalian Naval Academy, Dalian 116000, China; \\ ${ }^{2}$ Department of Basis, Dalian Naval Academy, Dalian 116000, China; \\ ${ }^{3}$ School of Foreign Languages, Shandong Normal University, Jinan 250000, China \\ a343065787@qq.com, bhongmei_005@163.com, 'czeternalx@163.com
}

Keywords: Higher Mathematics; MATLAB; Teaching application; Three-dimensional simulation.

\begin{abstract}
As a public basic required course of college, status of Higher Mathematics is of vital significance. However, the theory is too difficult to understand, most students think that math is useless so that they are not interested in higher mathematics. Today, MATLAB is one of the most developed software packages available. The purpose of this paper is to enable most students and particularly non-math majors to understand how to use MATLAB correctly in solving their problems of interest. In this paper, we focused on understanding the fundamental mathematical concepts and mastering problem-solving skills with the help of MATLAB and skip some tedious derivations. We found that by taking the MATLAB as an auxiliary and applying it into the teaching of higher mathematics, mathematical problems would be easy to solve. In this paper, almost every algorithm is followed by example MATLAB code with a friendly interface, so that students can easily modify the code to solve practical problems.
\end{abstract}

\section{Introduction}

Higher mathematics, a core course of science and engineering students in universities, provides essential mathematics basic knowledge and frequently-used methods for students learning later professional courses and solving practical problems. Ignoring the training of practical application, the Modern teaching methods of mathematics mainly focus on introducing of conception and definition, proving of theorem and practicing of calculating technics, which made students learning only for examination. Without using after study, students lack the understanding of mathematics application and scientific calculation methods [1].

With the development of computer technology and mathematics software, MATLAB has become a significant way of learning and researching in mathematics. By using it, students can accomplish drawing graphics, sign operation and numerical operation in mathematics all by themselves. What's more, MATLAB can visualize the abstract conceptions and theorem into the graphics [2]. Students learn from what they do, which makes them not only have a deeper understanding of basic conception and heighten their interests and confidence to learn mathematics, but also stimulate their desire of innovation. Besides, it also promotes the transformation from theory to practice.

\section{Features of MATLAB}

MATLAB [3], the abbreviation of Matrix Laboratory, as the business mathematics software produced by Math Works, is the most popular and the most widely used Engineering calculation and simulation software. Among many science and technology application software in mathematics, MATLAB comes first on the list in numerical operation. It has many functions, for instance, numerical analysis, graph and data processing, sign operation, word processing, mathematical modeling, real-time control, dynamic simulation and signal processing and so on.

\subsection{Powerful mathematic functions.}

There is amount of computational algorithm in Math Operations Library, including basic functions (trigonometric function, complex operation etc.), matrix operation (orthogonal decomposition, 
trigonometric decomposition, eigenvalue, determinant etc.), various special functions (Bessel function, Legendre function, Gamma function, Beta function, Elliptic function etc.) and all kinds of numerical operation, for example, numerical differentiation, numerical integration, extreme value, ordinary differential equation solver and so on.

\subsection{Powerful Graphics processing systems.}

By using MATLAB, 2-D and 3-D graphs can be drawn expediently and the function of image processing can be achieved. What's more, with the help of MATLAB, graphics can be added to marks and printed. There is also Icon Editor GUI in MATLAB, such as uicontrol and uimenu, users can accomplish satisfied graphical interface according to their needs.

\subsection{Powerful toolboxes.}

There is a dedicated family product called Toolbox in MATLAB and it used for solving problems in different areas. For example, Curve Fitting Toolbox, Optimization Toolbox, Statistics Toolbox and so on.

\subsection{Simple programming language.}

MATLAB is a kind of senior programming language based on matrix and array [4]. There are many features that need to be considered, such as program flow control, functions and data structure. Users use it to write simple programs quickly as well as complicated application programs.

\subsection{Formidable extended function.}

API is a function library that builds the connection between MATLAB, FORTAN and other high-level programming language like $C$. Users can take MATLAB as the calculation engine in FORTRAN or C.

\section{Higher Mathematics Application}

As a powerful calculating tool, MATLAB has an extensive application in mathematics operation. It is of help for students learning it well by taking the MATLAB as an auxiliary and applying it into the teaching of higher mathematics. Now, we will show you the important auxiliary function of MATLAB in higher mathematics by evaluation of differential equations, evaluation of multiple integrals, evaluation of the sum of Series, extreme value of functions of several variables, and creating 3-D graphs [5].

\subsection{Evaluation of Differential Equations.}

MATLAB supplies many tools to solve differential equations. Generally, dsolve() function can be taken to get the general solution and particular solution of Differential Equations. Functions and descriptions are shown in Table 1.

Table 1 Evaluation of Differential Equations

\begin{tabular}{cc}
\hline Function & Description \\
\hline$y=$ dsolve('eq') & general solution \\
$y=$ dsolve ('eq ', 'cond1', 'cond2',...' $v$ ') & particular solution \\
cond1,cond2: \\
initial condition \\
$y=$ dsolve('eq1', 'eq2',...' cond1', 'cond2',...' $v$ ') & particular solution \\
\hline
\end{tabular}

Example 1. Finding the general solution and particular solution of the following differential equation,

$$
y^{\prime}+\frac{1}{x} y=\frac{\sin x}{x}, y(\pi)=1
$$

Solution:

Type in the MATLAB Command window as below:

>> clear;clc;

syms $\mathrm{x}$;

$y 1=$ dsolve $\left({ }^{\prime} D y+y / x=\sin (x)^{\prime},{ }^{\prime} x\right)$; $\quad \%$ general solution

$\mathrm{y} 1=\operatorname{sinple}(\mathrm{y} 1)$ 
y2=simple(dsolve( 'Dy+y/x=sin(x)','y(pi)=1','x')) \% particular solution

The results are

$$
y_{1}=\frac{C_{2}}{x}-\frac{\cos x}{x}, y_{2}=\frac{\pi-\cos x-1}{x} .
$$

\subsection{Evaluation of Multiple Integrals.}

Generally, we can use int() function to solve multiple integrals. Functions and descriptions are shown in Table 2.

Table 2 Evaluation of Multiple Integrals

\begin{tabular}{cc}
\hline Function & Description \\
\hline$I=\operatorname{int}\left(\operatorname{int}\left(f, x, x_{1}, x_{2}\right), y, y_{1}, y_{2}\right)$ & $\int_{y_{1}}^{y_{2}} \mathrm{~d} y \int_{x_{1}}^{x_{2}} f(x, y) \mathrm{d} x$ \\
$I=\operatorname{int}\left(\operatorname{int}\left(\operatorname{int}\left(f, x, x_{1}, x_{2}\right), y, y_{1}, y_{2}\right), z, z_{1}, z_{2}\right)$ & $\int_{z_{1}}^{z_{2}} \mathrm{~d} y \int_{y_{1}}^{y_{2}} \mathrm{~d} y \int_{x_{1}}^{x_{2}} f(x, y, z) \mathrm{d} x$
\end{tabular}

Example 2. Please resolve the following triple integrals: $I=\iiint_{\Omega} z \mathrm{~d} x \mathrm{~d} y \mathrm{~d} z$,

$\Omega$ is a closed region bounded by $z=\sqrt{2-x^{2}-y^{2}}$ and $z=x^{2}+y^{2}$.

Solution:

(1) The projection

$$
\left\{\begin{array}{l}
x^{2}+y^{2}=\sqrt{2-x^{2}-y^{2}} \\
z=0
\end{array} .\right.
$$

Plot the integration region and its projection in the XY-plane, they are shown in Fig. 1.
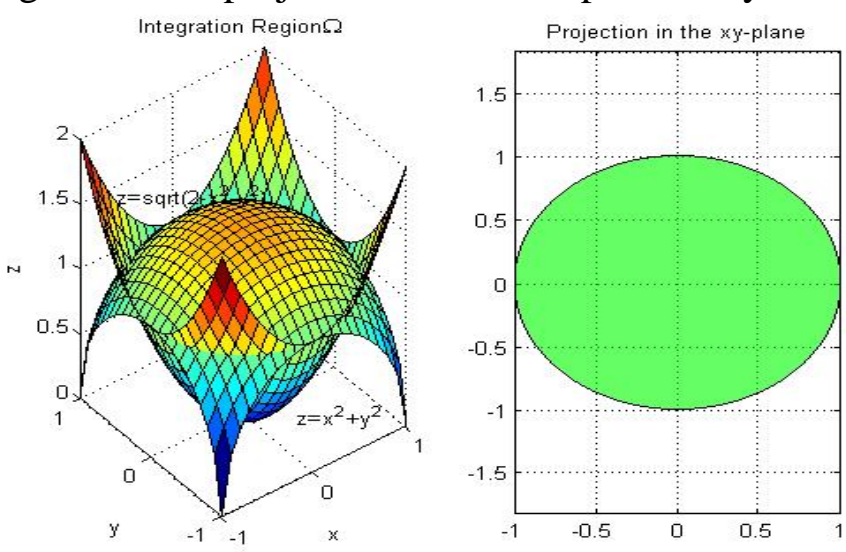

Fig. 1 Integration region and its projection in the XY-plane

(2) Evaluation of triple integrals by using cylindrical coordinates, the integration region is

$$
\Omega:\left\{\begin{array}{l}
\rho^{2} \leq z \leq \sqrt{2-\rho^{2}} \\
0 \leq \rho \leq 1 \\
0 \leq \theta \leq 2 \pi
\end{array} .\right.
$$

Type in the MATLAB Command window as below:

>> syms $r$ theta

$\mathrm{f}=\mathrm{z} ; \mathrm{r} 1=0 ; \mathrm{r} 2=1 ; \mathrm{t} 1=0 ; \mathrm{t} 2=2 * \mathrm{pi}$;

$\mathrm{x}=\mathrm{r}^{*} \cos ($ theta); $\mathrm{y}=\mathrm{r} * \sin ($ theta);

$\mathrm{z} 1=\operatorname{sprt}\left(2-\mathrm{x}^{\wedge} 2-\mathrm{y}^{\wedge} 2\right) ; \mathrm{z} 2=\mathrm{x}^{\wedge} 2+\mathrm{y}^{\wedge} 2$;

$\mathrm{I}=\operatorname{int}(\operatorname{int}(\operatorname{int}(\mathrm{f} * \mathrm{r}, \mathrm{z}, \mathrm{z} 2, \mathrm{z} 1) \mathrm{r}, \mathrm{r} 1, \mathrm{r} 2)$,theta,t1,t2)

The result is $I=\frac{7 \pi}{12}$. 


\subsection{Application of Series.}

Series is an important form of functions in Higher Mathematics. Many complicated functions can be showed by Series [3]. There's symsum() function to solve the sum of the series. Functions and description are shown in Table 3.

Table 3 Sum of the series

\begin{tabular}{cc}
\hline Function & Description \\
\hline$s=\operatorname{symsum}\left(u_{n}, n, a, b\right)$ & $\sum_{n=a}^{b} u_{n}$ \\
\hline
\end{tabular}

Example 3.Please solve the following series $\sum_{n=0}^{50}\left[a n^{3}+(a-1) n^{2}\right]$.

Solution:

We can use the symsum() function to obtain the sum of an indefinite/definite series as below:

$>>\mathrm{f}=\mathrm{a} * \mathrm{n} \wedge 3+(\mathrm{a}-1) * \mathrm{n} \wedge 2$;

$\mathrm{s}=\operatorname{symsum}(\mathrm{f}, \mathrm{n}, 0,50)$

The result is: $s=-42925+1998550 a$.

\subsection{Extreme Value of functions.}

There're many functions to solve the extreme value of functions [3]. Functions and description are shown in Table 4.

Table 4 Extreme Value of functions

\begin{tabular}{cc}
\hline Function & Description \\
\hline$[x, \mathrm{fval}]=$ fminsearch $\left(f, x_{0}\right)$ & We can use these functions to get the local minimum. \\
{$[x, \mathrm{fval}]=\mathrm{fminunc}\left(f, x_{0}\right)$} & If you want to obtain the local maximum, you can \\
\end{tabular}

Example 4. Finding the extreme value of $f(x, y)=x^{3}-y^{3}+3 x^{2}+3 y^{2}-9 x$.

Solution:

We can use the fminsearch() function to get the extreme values of the function as below:

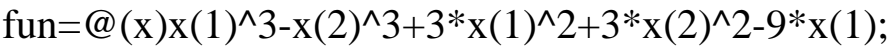

[xmin,fmin] $=$ fminsearch(fun,rand(1,2)) \% local(relative) minimum

fun=@(x)-fun(x);

[xmax,fmax $]=$ fminsearch(fun,rand(1,2)) \% local(relative) maximum

Then, the local minimum is $f(1,0)=-5$, local maximum is $f(-3,2)=31$.

\subsection{Creating 3-D Graphs.}

Nowadays, space analytic geometry in the textbooks all plane figures, which is difficult for students to build the conception of space figures [3]. With the help of MATLAB, 3-D Graphs can be easily displayed with animation and the knowledge can be expressed in a vivid way. The result is that students build up the ability of space imagination easier. Doesn't it embody the combination of theory and practice?

Example 5. Please plot the intersection curve of such two space surfaces, $z=x^{2}-2 y^{2}$ and $z=a$.

Solution:

The parametric equations of curve:

$\left\{\begin{array}{l}x=1+\cos t \\ y=\sin t \\ z=2 \sin t / 2\end{array}\right.$.

These statements produce the intersection curve:

[X,Y,Z]=sphere(60);

$\operatorname{surf}(2 * \mathrm{X}, 2 * \mathrm{Y}, 2 * \mathrm{Z}) ; \quad \%$ Plot a sphere

hold on 

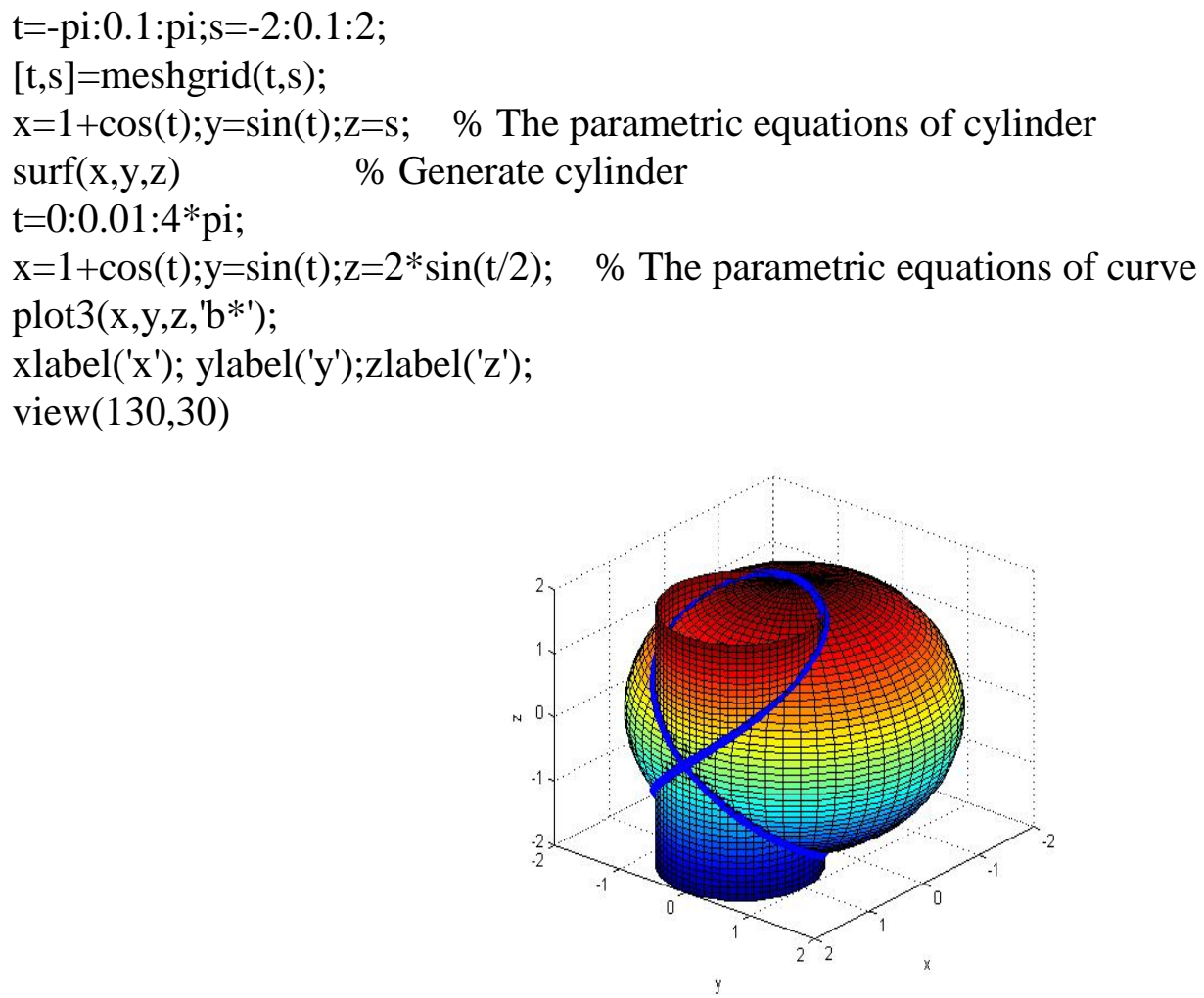

Fig. 2 Intersection curve

\section{Conclusion}

In this paper, we discuss several basic applications of MATLAB in higher mathematics, including evaluation of differential equations, multiple integrals, the sum of Series, extreme value of functions of several variables, and creating 3-D graphs. Higher mathematics education is an important part in training students' mathematics quality [6], we found that by taking the MATLAB as an auxiliary and applying it into the teaching of higher mathematics, mathematical problems would be easy to solve. This process will help students not only to acquire the basic knowledge and skill but also strengthen their creative abilities [7]

\section{References}

[1]. Wang Biao. Brief Analysis on Application of MATLAB in Higher Mathematics Teaching*[J]. Computer \& Digital Engineering, 2013, 8:1357-1359.

[2]. Zhenlin Wang, Ming Liu. Proficient in MATLAB (updated version) [M]. Beijing: Publishing House of Electronics Industry, 2011.1.(In Chinese)

[3]. Yaqun Huang. Higher Mathematics Experiments based on MATLAB [M]. Beijing: Publishing House of Electronics Industry, 2014.8.(In Chinese)

[4]. Won Young Yang, Wenwu Cao, Tae-Sang Chung, John Morris. Applied Numerical Methods Using MATLAB®. John Wiley \& Sons, Inc., 2005:321-370.

[5]. Weiliang Zeng, Liu Ying. Three-dimensional simulation function of MATLAB in the teaching practice of mathematical model course [J].Natural science journal of Harbin Normal University, 2006:3(22).

[6]. Liu Jun. Design and Development of network Curriculum of Higher Mathematics [D]. Tianjin: Tianjin Normal University, 2012. 
[7]. Jiuhong Zeng. Research and Practice of Cultivation of Students Mathematics Application Ability in Higher Mathematics Teaching [D]. Hunan: Hunan Normal University, 2012. 